\title{
Foraging strategies of the marine iguana, Amblyrhynchus cristatus
}

\author{
Krisztina G.K. Trillmich and Fritz Trillmich \\ Max-Planck-Institut für Verhaltensphysiologie, Abteilung Wickler, 8131 Seewiesen, Federal Republic of Germany
}

Received March 15, 1985 / Accepted September 4, 1985

Summary. Two foraging strategies were found in marine iguanas (Amblyrhynchus cristatus); (1) subtidal feeding: the animals swam out to sea and dived for algae in the subtidal zone; (2) intertidal feeding: the animals foraged around low tide in the intertidal zone on more or less exposed algae. Most marine iguanas were very consistent in their foraging strategy and so could be classified as subtidal feeders (SFs) or intertidal feeders (IFs). Feeding strategy was weight-related (Fig. 1), not sexspecific. Animals $<1,200 \mathrm{~g}$ were IFs, animals $>1,800 \mathrm{~g}$ SFs. Some iguanas in between followed a mixed foraging strategy. SFs foraged independently of the tides, IFs always around low tide (Figs. 2, 3). Feeding time patterns of IFs and SFs are described (Table 1). Sea motion seemed to have little effect on the foraging pattern of SFs, but strongly influenced that of IFs (Fig. 2). The smaller a marine iguana, the faster it cooled when immersed in water (Fig. 4). The difference between water temperature and core temperature of animals returning from foraging was significantly greater in IFs than SFs (Fig. 5). SFs swimming in very cold water regulated their body temperature to prevent excessive cooling. Possible costs and benefits of the two foraging strategies are discussed. Only part of a marine iguana population lives really amphibiously and only ca. $5 \%$ of a $24 \mathrm{~h}$ day is spent close to or in the water. All social activities, including mating, take place on land. These life history characteristics preclude those adaptations to an amphibious way of life that would at the same time reduce the iguanas' ability to be maximally active at their typical terrestrial body temperature of $35^{\circ} \mathrm{C}$.

\section{Introduction}

Differences in foraging strategies within a population have been described for mammals (Gentry
1981), birds (Newton 1979; Peters and Grubb 1983), several large reptiles (Cott 1961; Auffenberg 1972) and many amphibians, fish and invertebrates (Werner and Gilliam 1984). In mammals and birds these differences usually correlate with size or social dominance differences between the sexes. But amongst reptiles, ontogenetic size development seems to be most important (Pough 1973; Pond 1977). Reptilian young must feed themselves after hatching. As body size strongly influences the type of food resources which can be efficiently exploited, young reptiles have to pass through several niches as they grow. This also applies to Galapagos marine iguanas (Amblyrhynchus cristatus) which hatch at about $48-65 \mathrm{~g}(n=4)$; males on Fernandina grow to a mass of about $5 \mathrm{~kg}$, a roughly 70-fold increase in body mass.

Marine iguanas feed almost exclusively on algae. Darwin (1883), Hobson (1965), Carpenter (1966) and Boersma (1984) reported most feeding at low tide in the exposed intertidal zone, and in the subtidal zone where the animals dive to reach algae. Bartholomew (1966) found that feeding activity occurred throughout the day and at any stage of the tide. Some studies also mention differences in foraging behavior of various age/sex classes. Bartholomew et al. (1976) found that juveniles kept to shallow water and only adults fed additionally in subtidal areas. Boersma (1984) suggested that females foraged in the intertidal and males in subtidal zones. These partly conflicting observations point to sex and/or age specific foraging strategies within a given marine iguana population.

Marine iguanas have few special morphological and physiological adaptations for dealing with an amphibious way of life. Swimming and diving abilities and cardiovascular mechanisms for heat retention resemble those of typical terrestrial reptiles (Bartholomew and Lasiewski 1965; Bartholomew et al. 1976; Bennett et al. 1975; Dawson et al. 1977). Thus behavioral mechanisms must be vital 
in allowing them to use their exceptional food resource efficiently enough to sustain their very large colonies.

This study examines the foraging strategies of medium to large marine iguanas, and the intrinsic and ecological factors which determine foraging strategy choice.

\section{Methods}

Marine iguana foraging was observed in detail in September/ October 1976 on Caamaño, a small islet in Academy Bay, Santa Cruz, and between August and November 1977 at Cabo Hammond, Fernandina, where only the approximate times of day of marine iguana intertidal feeding were also noted in August/ September 1979 and September/October 1983. Data on foraging patterns during the reproductive season were not used, as territorial males forage much less (Trillmich 1983).

Animals were noosed or caught by the tail for marking. Captured animals were sexed (reliable only when $>$ approx. $500 \mathrm{~g}$ ), weighed to the nearest 10 or $50 \mathrm{~g}$ on spring balances and marked with numbered plastic tags and painted numbers on their flanks (Trillmich 1983).

Marine iguanas on Caamaño were observed for 6 weeks. Fourteen marked large males (range 1,800-4,700 g) were observed continuously for 14 days during this period. Movements to and from the sea, and resting ashore outside the colony were recorded. Whenever the exact time of a move was missed, the sequence was not used for analysis of the feeding pattern.

On Fernandina most of the 60 marked individuals (range $500-5,250 \mathrm{~g}$ ) belonged to one colony. Their presence in the colony was checked several times a day. For classification as subtida1 or intertidal feeders animals were noted on 32 days whenever they were observed swimming in the subtidal or foraging in the intertidal zone. As the marked intertidal feeders foraged exclusively in a roughly $100 \mathrm{~m}$ section of coastal intertidal, they could be surveyed comprehensively by one or two observers. A $170 \mathrm{~m}^{2}$ area (study area) of this section, clearly demarcated and in plain view, was chosen for continuous observation on 20 consecutive days of the 32 days. Duration of foraging bouts and pauses between bouts were noted for all marked animals seen there. Seven fed almost daily in the study area permitting comparison of their foraging behavior on different days.

Including observations on unmarked animals we recorded: (1) presence or absence of any feeding activity on a given day, (2) times of day of intertidal or subtidal feeding, (3) duration of the intertidal foraging period (i.e. when any animal was seen feeding in the study area), and (4) number of animals feeding in the study area, counted every 5 min during a foraging period.

Sea surface temperature near the colony was measured daily and sea condition rated calm, normal or rough: frequent flooding of the intertidal by high waves around low tide was defined as rough.

Changes in body temperature of 6 animals upon immersion in water were measured on Fernandina. Warm animals were captured in the colony, weighed and their temperature measured with a thermoprobe inserted at least $2 \mathrm{~cm}$ into the cloaca (up to $6 \mathrm{~cm}$ for larger animals). Temperature was read from an analog indicator (BBC-Metrawatt P500) to the nearest $0.1^{\circ} \mathrm{C}$. Iguanas were tethered and lowered into the water by a nylon line attached to a pole. They were lifted out of the water for a few seconds every 5 min to measure core temperature, then immediately submerged again. Experiments lasted 30-60 min until core temperature was close to sea surface temperature. Core temperature and mass of 44 marine iguanas re- turning from undisturbed foraging bouts in the intertidal or subtidal were also measured.

\section{Results}

\section{Foraging strategies}

Initial qualitative observations showed that the marine iguanas used 2 foraging strategies:

1. Subtidal feeding. Animals using this strategy swam out to sea daily, or every 2 or 3 days, and made several dives in the subtidal zone to feed on submerged algae. They often swam about $400 \mathrm{~m}$ along the coast to subtidal areas further from the colony. Single dives lasted from 60 to $815 \mathrm{~s}$, with a median of $175 \mathrm{~s}(n=23$, observations on 11 individuals; compare Bartholomew et al. 1976; Carpenter 1966; Hobson 1969). Between dives animals swam short distances or swam back and forth close to where they had last dived. After a foraging bout at sea (measured from entering to leaving the water) they sometimes sprawled on sunlit rocks to warm up, before starting another foraging bout or returning to the colony, walking on land or swimming along the coast.

2. Intertidal feeding. Animals using this strategy made daily excursions around low tide into the intertidal zone near the colony to forage on more or less exposed algae. They sometimes submerged completely in tidepools, swam across small channels or were washed by breakers. Intertidal feeders foraged continuously for a long time, then returned to the colony, or rested between foraging bouts (time spent feeding in the intertidal zone), sprawled on sunny wind-protected rocks in the intertidal.

\section{Individual consistency in the use of foraging strategies}

Marked marine iguanas were very consistent in their foraging strategy. Sixty animals were regularly observed, 46 on Fernandina and 14 on Caamaño. Nineteen were each observed foraging at least 13 times (median 92 times) always in the intertidal, never at sea. A further 3 animals, each observed at least 33 times, swam in less than 5\% of all observations but only very close to the intertidal. All these were classed as intertidal feeders (IF).

Twentyfive iguanas (14 on Caamaño) foraged in the subtidal zone, but were never observed feeding in the intertidal. They were called subtidal feeders (SF).

Thirteen animals fed in both zones. Four of 


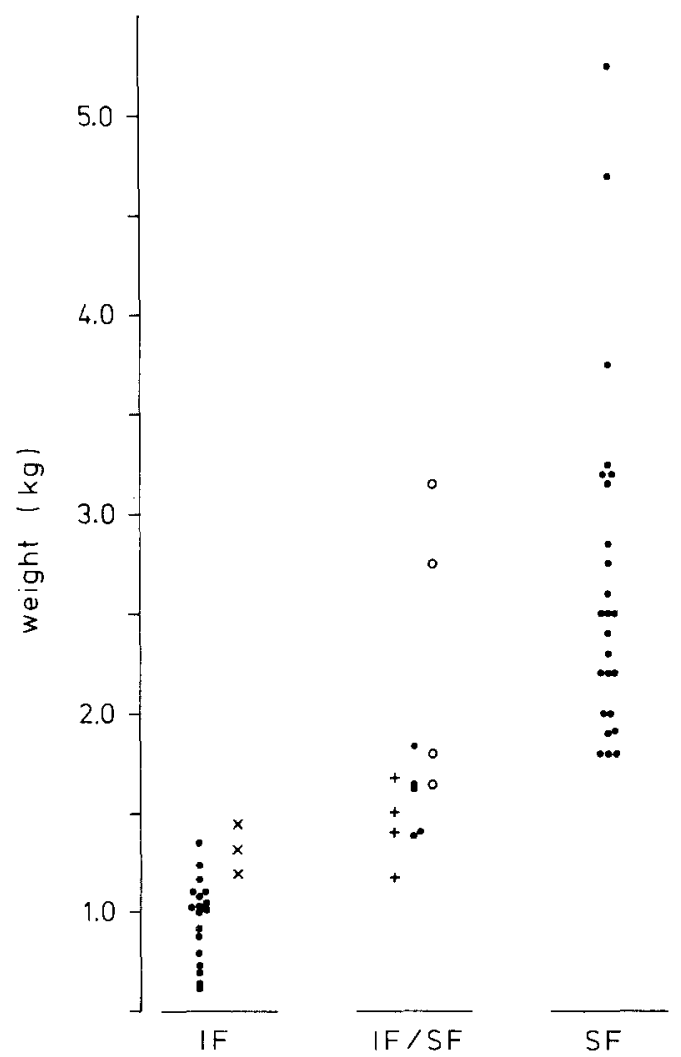

Fig. 1. Feeding strategy is weight-related. $I F=$ intertidal feeders. $\mathrm{SF}=$ subtidal feeders. $\mathrm{IF} / \mathrm{SF}=$ animals with a mixed strategy. $\mathrm{x}=$ animal in $<5 \%$ of observations swimming, always very close to the intertidal. $+=$ in $<20 \%$ of observations swimming. $\mathrm{o}=$ in $>80 \%$ of observations swimming

these behaved more like IFs: they swam in less than $20 \%$ of all observations (15-125 observations per animal with 2-16 individual records of swimming). Another 4 behaved more like IFs, foraging in the intertidal in less than $20 \%$ of the observations (6-34 observations per animal with 1-6 individual records in the intertidal). Thus only $5(8 \%)$ of 60 marked iguanas were regularly seen foraging in both zones.

\section{Foraging strategy is mass-related}

The foraging strategy of marine iguanas was related to body mass and not to sex. Animals weighing less than ca. 1,200 $\mathrm{g}$ belonged to the IFs, almost all animals above ca. 1,800 g to the SFs (Fig. 1). Iguanas in the intermediate $600 \mathrm{~g}$ range tended to follow a mixed foraging strategy.

The heaviest marine iguana female measured on Fernandina weighed $1,840 \mathrm{~g}$, the heaviest male $5,250 \mathrm{~g}$. The heaviest female on Caamaño weighed only $1,200 \mathrm{~g}$, the heaviest male $4,700 \mathrm{~g}$. This sexual size dimorphism explains why most animals seen
Table 1. Foraging of subtidal feeders (SFs) and intertidal feeders (IFs) on Caamaño and Fernandina. Caamaño data stem from $13 \mathrm{SFs}$, Fernandina data from $7 \mathrm{SFs}$ and 7 IFs. Days with no feeding activity were excluded. Total foraging time: total time an individual spent foraging on a given day; excludes travelling time between colony and sea (SFs) or intertidal (IFs) and time spent resting on land or in dry areas of the intertidal between foraging bouts

\begin{tabular}{|c|c|c|c|}
\hline & $\begin{array}{l}\text { SF- } \\
\text { Caamaño }\end{array}$ & $\begin{array}{l}\text { SF- } \\
\text { Fernandina }\end{array}$ & $\begin{array}{l}\text { IF- } \\
\text { Fernandina }\end{array}$ \\
\hline \multicolumn{4}{|c|}{$\begin{array}{l}\text { Foraging bout } \\
\text { length (min) }\end{array}$} \\
\hline $\begin{array}{l}\text { mean } \\
\text { median } \\
\text { range }\end{array}$ & $\begin{array}{l}33 \pm 8 \\
32 \\
5-69\end{array}$ & $\begin{array}{l}40 \pm 17 \\
44 \\
10-69\end{array}$ & $\begin{array}{l}36 \pm 32 \\
25 \\
2-169\end{array}$ \\
\hline$n$ & 13 & 15 & 216 \\
\hline \multicolumn{4}{|c|}{$\begin{array}{l}\text { Total foraging } \\
\text { time/day (min) }\end{array}$} \\
\hline $\begin{array}{l}\text { mean } \\
\text { median } \\
\text { range }\end{array}$ & $\begin{array}{l}52 \pm 8 \\
52 \\
10-103\end{array}$ & $\begin{array}{l}75 \pm 18 \\
77 \\
50-100\end{array}$ & $\begin{array}{l}70 \pm 36 \\
68 \\
7-169\end{array}$ \\
\hline$n$ & 13 & 8 & 118 \\
\hline \multicolumn{4}{|c|}{$\begin{array}{l}\text { Mean no. of foraging } \\
\text { bouts/day }\end{array}$} \\
\hline \multicolumn{4}{|c|}{$\begin{array}{l}\text { Length of foraging } \\
\text { pauses (min) }\end{array}$} \\
\hline $\begin{array}{l}\text { mean } \\
\text { median } \\
\text { range }\end{array}$ & $\begin{array}{l}41 \pm 8 \\
39 \\
13-107\end{array}$ & $\begin{array}{l}58 \pm 13 \\
59 \\
40-69\end{array}$ & $\begin{array}{c}39 \pm 12 \\
38 \\
6-79\end{array}$ \\
\hline$n$ & 13 & 7 & 95 \\
\hline \multicolumn{4}{|c|}{$\begin{array}{l}\text { Total time/day } \\
\text { foraging }+ \text { pauses }\end{array}$} \\
\hline $\begin{array}{l}\text { mean } \\
\text { median } \\
\text { range }\end{array}$ & $\begin{array}{l}77 \pm 15 \\
74 \\
10-185\end{array}$ & $\begin{array}{l}125 \pm 49 \\
137 \\
50-185\end{array}$ & $\begin{array}{l}105 \pm 47 \\
111 \\
\quad 7-208\end{array}$ \\
\hline$n$ & 13 & 8 & 104 \\
\hline
\end{tabular}

in the subtidal zone were males ( 30 males: $8 \mathrm{fe}-$ males, Fig. 1). The sex ratio of marked animals classed as IFs (15 females: 7 males, Fig. 1) matched the sex ratio in our sample of marked animals weighing $<1,500 \mathrm{~g}$ (17 females:9 males). Thus small animals of both sexes were intertidal feeders.

Very small animals $(<500 \mathrm{~g})$ were seen only between lava rocks and in small crevices in the spray zone, where they fed on low algal growth. They never ventured into the water to approach more distant foraging areas.

\section{IFs' and SFs' feeding time patterns}

Feeding time patterns were studied to obtain a detailed time budget for the two foraging strategies 

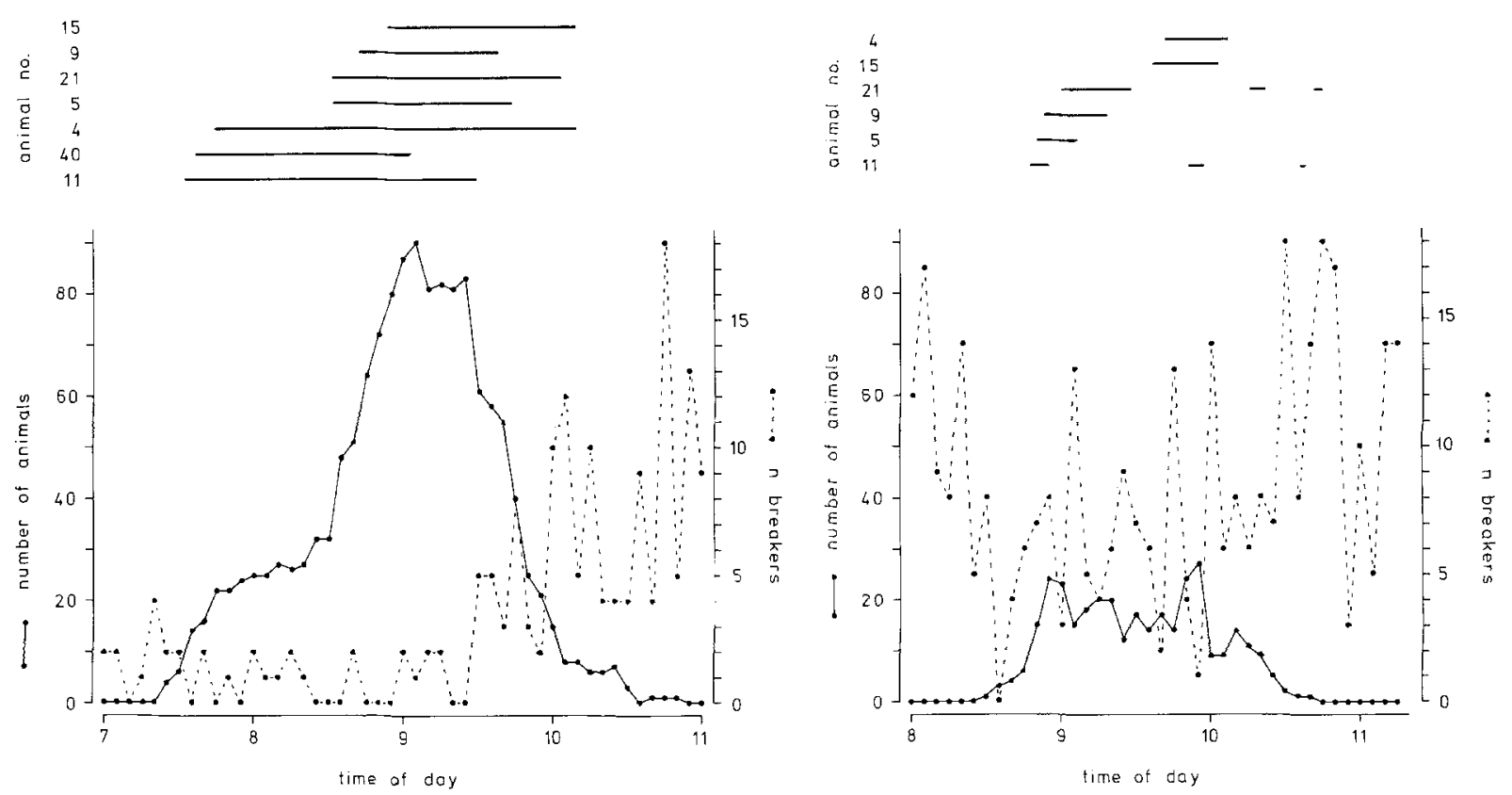

Fig. 2. Comparison of IF feeding pattern on a calm day (left: 9/27/77) and on a rough day (right: 9/28/77). Graph top: foraging bouts of numbered individuals. bottom: number of animals in the intertidal (from counts every 5 min) and number of breakers flushing the intertidal within 5-min intervals

(Table 1). IFs' foraging bouts and pauses were roughly as long as those of SFs on Caamaño and Fernandina. IFs' total daily foraging time was about as long as that of SFs on Fernandina $(P>$ $0.1 ; n 1=7, n 2=7$, Mann-Whitney $U$-test), but significantly longer than that of SFs on Caamaño $(P<0.001, n 1=7, n 2=13$, Mann-Whitney $U$-test $)$. Variances of foraging bout duration and of total daily foraging time for the most frequently observed matked individuals were larger in IFs than in SFs (both $P<0.001, n 1=7, n 2=13$, MannWhitney $U$-test). The IF-values in Table 1 were obtained over 20 days of continuous observation of 7 marked iguanas weighing $700-1,450 \mathrm{~g}$. They foraged up to 4 times per day, and fasted for a day 1-3 times per individual during the 20 day period. Inclusion of fasting days reduced the median of total foraging time per day to 63 min. Marked SFs on Caamaño made up to three foraging bouts per day, and fasted a day in only 9 out of 145 complete records of total foraging time. Inclusion of fasting days reduced the median of SFs' total foraging time per day to $49 \mathrm{~min}$.

\section{Influence of sea conditions on feeding}

Sea motion seemed to have little effect on the foraging pattern of SFs but strongly influenced that of IFs (Fig. 2), thus causing the high variance of the IFs' foraging bout duration and total foraging time (Table 1). The intertidal area was washed by strong breakers on days with heavy swell. Some iguanas were caught off guard and had to struggle against the backwash and many ran towards land between large waves. The animals made short foraging bouts under these conditions, but went into the intertidal up to 4 times a day (Fig. 2). Sometimes they fasted for a day when conditions were particularly bad. Total foraging times of marked IFs under rough conditions were significantly shorter than those in calm conditions (Randomization test, $P=0.015 ; n=6$ ) and those of "normal" days $(P=0.016 ; n=6)$.

Maximal numbers of animals (counts every 5 min, see Methods) and cumulative totals of all IF counts in the intertidal were significantly lower on rough than on calm days (Mann-Whitney $U$ test, $n 1=5, n 2=6 ; P=0.004$ and $P=0.002$ ) and correlated significantly with the average total foraging time of marked individuals $(r=0.78, P<$ $0.001, n=19$, and $r=0.87, P<0.01, n=19$ ). The lower maximal numbers on rough days suggest also that fewer animals foraged in the intertidal when conditions were difficult.

\section{Timing of foraging in relation to the tides}

Subtidal and intertidal foraging were differently influenced by the tidal cycle. SFs foraged at some time between late morning and early afternoon, relatively independently of the tides (Fig. 3).

IFs could reach their food supply only at low 


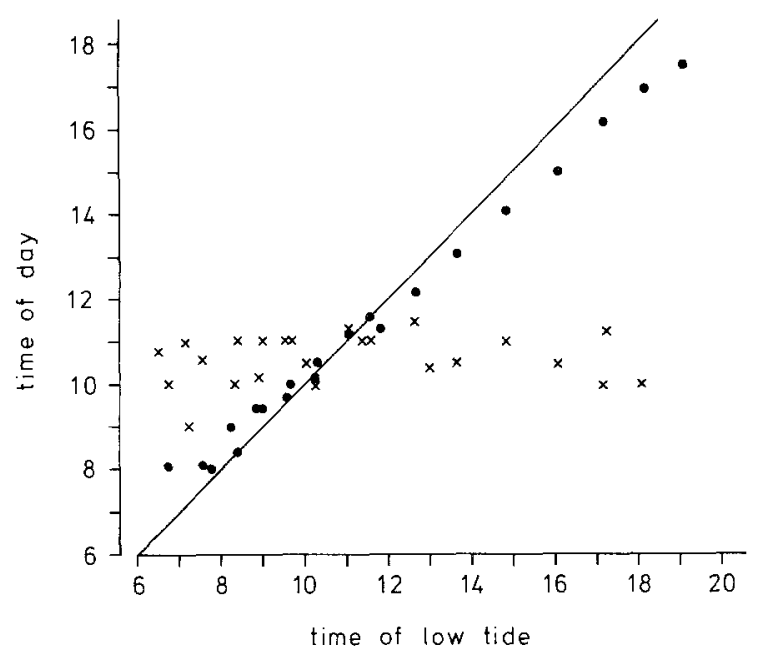

Fig. 3. SFs (x) forage independently of the tide. The graph shows the time when first SFs swam out to forage (data from 25 days) plotted against the time of low tide. IFs ( ) forage around low tide. Median foraging time is plotted against the time of low tide. As long as marine iguanas were foraging in the intertidal their numbers were counted every $5 \mathrm{~min}$. Median foraging time is defined as the time when $50 \%$ of the sum of all counts was reached during one low tide. The diagonal is the hypothetical line for the coincidence of ebb and foraging

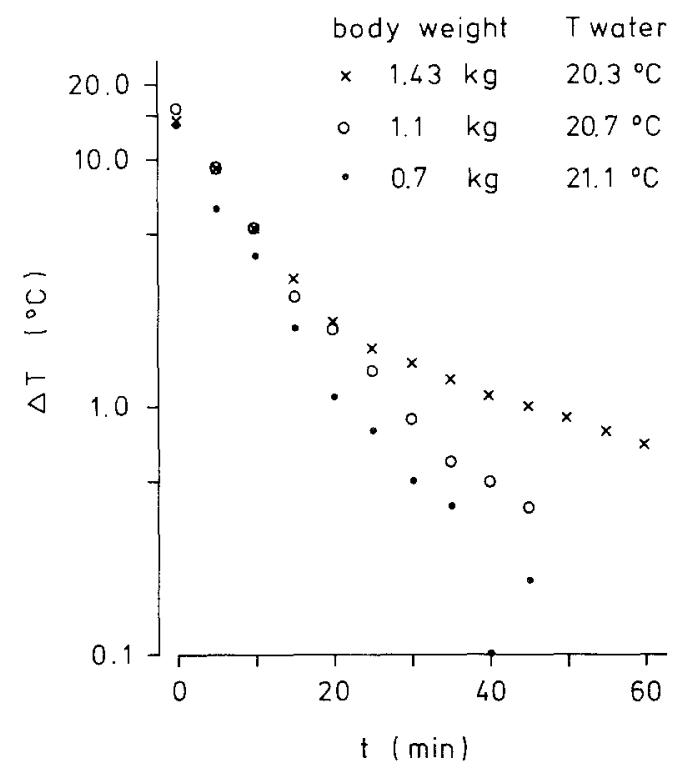

Fig. 4. Cooling rates of marine iguanas of different weights. $\Delta T=$ difference between core temperature of the marine iguana and water temperature

tide, which is about $50 \mathrm{~min}$ later daily. Marine iguanas live on the equator in a $12: 12 \mathrm{~h}$ light: dark cycle. So IFs should forage once per day, unless two ebbs fall during daylight (early morning and evening).

This was found to be so. The daily foraging period lasted $3 \mathrm{~h} 20 \mathrm{~min}$ (mean and median, $n=20$; range $2 \mathrm{~h} 10 \mathrm{~min}$ to $4 \mathrm{~h} 30 \mathrm{~min}$ ). IFs' foraging was closely coupled to the shift of the low tide, i.e. a little later each day (Fig. 3), as expected. However, low tide and median feeding time (when $50 \%$ of the cumulative total of IFs had been counted in the intertidal) were not perfectly synchronous. Median feeding time in the morning lagged behind low tide, in the afternoon it preceded it.

IFs did not feed after dusk and only up to 2-3 $\mathrm{h}$ in advance of low tide. They switched foraging from evening to morning when low tide was after ca. 18.30 hours. The latest low tide at which IFs fed exclusively in the evening was at 18.48 hours; the earliest equivalent morning low tide was at 05.52 hours.

IF feeding activity was observed to switch from evening to morning low tide on 12 occasions (1 in 1976, 4 in 1977, 1 in 1978, 2 in 1979, and 4 in 1983). Feeding in both evening and morning was noted only 4 times. The interval between switches was 13,14 and 15 days $(n=6)$, following a semilunar rhythm.

\section{Cooling : experimental and natural}

The smaller a marine iguana, the faster it cooled when immersed in water (Fig. 4 and Bartholomew 1966). One would therefore expect the smaller IFs to cool down more than SFs during foraging. But the difference between the water temperature and core temperature $(\Delta T)$ of IFs returning from foraging on Fernandina was significantly greater than in returning SFs $(P<0.001 ; n 1=15, n 2=24$, Mann-Whitney $U$-test; water temperatures 16.5-21. $3^{\circ} \mathrm{C}$ (Fig. 5). This result is not an artifact due to measurement of animals at different water temperatures. The same result was obtained comparing IF and SF subsamples at very similar water temperatures $\left(20.2-21.3^{\circ} \mathrm{C} ; \quad P<0.001 ; n 1=9\right.$, $n 2=12)$. Nor is the difference the effect of the negative correlation between $\Delta T$ and body mass $(r=$ $-0.55, P<0.01, n=44)$. If it were we would expect to find the same negative correlation within the groups of SFs and IFs, which is not the case (SF: $r=-0.03$, NS; IF: $r=-0.19$, NS). Apparently IFs fed in an environment where they lost heat much more slowly than SFs and in which behavioral thermoregulation and heat gain from solar radiation made it easier for them to maintain a higher body temperature.

The body temperature of many SFs returning from foraging was barely above water temperature (Fig. 5). There was nevertheless some evidence of thermoregulation as the $\Delta T$ in SFs returning from foraging was significantly negatively correlated 


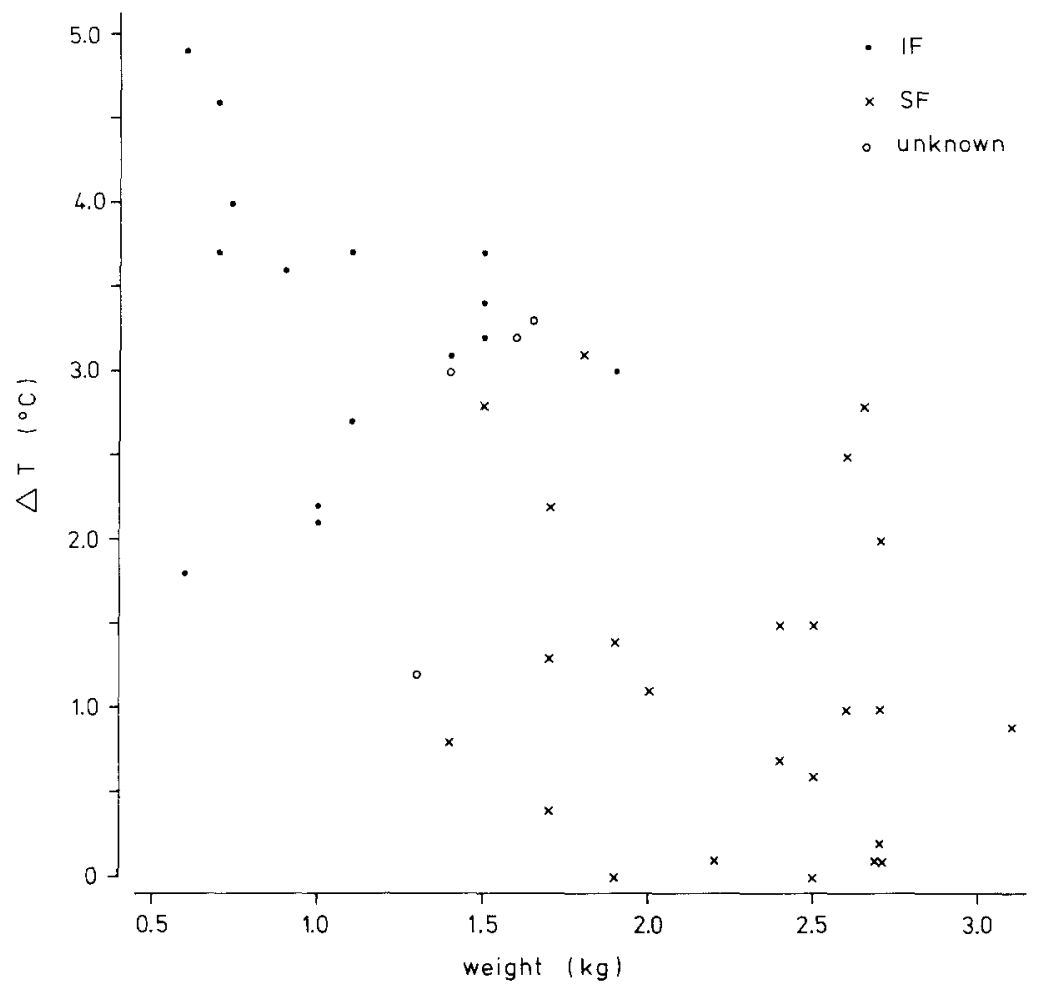

Fig. 5. Difference between water temperature and core temperature $(\Delta T)$ of marine iguanas returning from foraging is greater in the smaller IFs. Core temperature is almost always higher than water temperature with water temperature $(r=-0.78, P<0.01, n=$ $25)$. SFs swimming in very cold water regulated their body temperature by physiological or behavioral means (e.g. shorter foraging bouts) to prevent excessive cooling.

\section{Discussion}

Size, sex and the choice of a foraging strategy

Marine iguanas weighing $>1,800 \mathrm{~g}$ were SFs, those weighing $<1,200 \mathrm{~g} \mathrm{IFs}$, and some of intermediate weight followed a mixed strategy (Fig. 1). This relationship resolves the contradictions over foraging strategy choices in the literature (Bartholomew et al. 1976; Boersma 1984). Clearly, all females do not forage in the intertidal nor do all males feed by swimming and diving in the subtidal, as suggested by Boersma (1984). Rather juveniles and adults of both sexes below $1,200 \mathrm{~g}$ are IFs. The smallest marine iguanas (ca. $50-400$ or $500 \mathrm{~g}$ ) are - strictly speaking - not even intertidal feeders. Animals of either sex gradually substitute subtidal for intertidal foraging as they approach $1,800 \mathrm{~g}$. As males grow to a larger final size than females they form the majority of the SF group. This is typical for the situation at Cabo Hammond. However, the mass threshold at which individuals change their strategy may vary from island to island depending (1) on the size of the intertidal zone,
(2) on the abundance of food in the intertidal and subtidal areas, and (3) on the density of the iguana population.

\section{Why choose a particular foraging strategy?}

One obvious advantage of subtidal foraging is greater independence of weather conditions impeding intertidal foraging (Fig. 2). This is supported by the much lower variance in SFs' foraging bout duration and total foraging time per day, compared to those of IFs (Table 1). Although SFs had difficulties to cross the surf they gave up foraging only in much rougher conditions than IFs.

Subtidal foraging is independent of the tidal cycle, unlike intertidal foraging (Fig. 3). IFs have to match their foraging to the tides, and may have insufficient time to warm up before foraging on early morning or after foraging on late evening low tides. In the former case the animals have to interrupt feeding sooner to warm up. Evening foraging may leave them little time to warm up enough after foraging to assimilate food efficiently overnight (White 1973; Boersma 1982). These are no problems for SFs, which can forage in the late morning or around noon (Fig. 3), when solar radiation is more intense and heat stored in rocks permits rapid warm-up.

Two size specific constraints appear to limit subtidal foraging to large individuals: (1) marine 
iguanas are inferior swimmers compared to similar sized bony fish, and the smaller an iguana the more inefficient its swimming (Bartholomew et al. 1976; Vleck et al. 1981). Thus small iguanas could be swept away by even moderate currents. (2) Small iguanas lose heat faster than larger ones (Fig. 4). If they foraged in the subtidal their only possible behavioral thermoregulation would be to return ashore. Given their limited swimming ability this would mean increased travel costs and reduced feeding time compared to larger iguanas. Poor mobility at low body temperature may also expose small marine iguanas to a higher mortality risk on land through predators such as the Galapagos hawk. This may explain why IFs keep their body temperatures from falling as low as SFs (Fig. 5).

Behavioral regulation of body temperature is easier for foraging IFs as they remain within easy reach of dry rocks. Furthermore, cooling is much reduced as teir backs are almost constantly exposed to the sun, and periods of complete immersion are short.

One might expect that the larger animals would also benefit from foraging in the intertidal, due to reduced travel and thermoregulation costs. To explain why they forage subtidally, one may postulate different densities of food and/or of foragers in the two zones. The density of foraging iguanas at Cabo Hammond was higher in the intertidal. Algae there were usually grazed down, which must have reduced food intake per bite. Presumably the larger iguanas increased their food intake per unit time by swimming out and diving, thus avoiding the scramble competition with smaller conspecifics. Large iguanas are also able, at low cost, to survey wide areas for food patches, as their energetic cost of swimming per unit distance is $75 \%$ less than that of walking (Gleeson 1979).

\section{Why have marine iguanas not adapted to low operating temperatures?}

Marine iguanas lead a much less amphibious life than previously assumed. Only SFs are truly amphibious and derive the full benefits of reptilian pre-adaptations to this mode of life (Dawson et al. 1977). An estimated $50 \%$ of the Fernandina study colony and more than $80 \%$ of the Caamaño population were IFs. Thus the majority of individuals in the study populations are terrestrial either throughout life or a substantial part of their ontogeny.

Time spent per day close to or in the water (Table 1) averaged 76-125 min, including warming-up pauses between foraging bouts; the figure for total foraging time only was $52-75 \mathrm{~min}$. This amounts to roughly $5 \%$ of a $24 \mathrm{~h}$ day. Thus even the most amphibious large males spend about $95 \%$ of the time ashore. Only $7-8 \%$ of their daily energy budget is spent foraging (Nagy and Shoemaker 1984). It therefore seems logical that most of their adaptations are for a terrestrial way of life. However, Dawson et al. (1977) suggest that marine iguanas' most strenuous activities occur in water at body temperatures around $25^{\circ} \mathrm{C}$, and are surprised to find no clear signs of adaptation to hard exercise at this body temperature. But male and female activities on land during the reproductive season appear far more strenuous (Boersma 1984; Eibl-Eibesfeldt 1966; Trillmich 1983; Rauch, personal communication). Marine iguanas certainly cannot compromise their ability to be very active on land at body temperature of $35-37^{\circ} \mathrm{C}$ without jeopardizing successful reproduction. It appears to be very costly and difficult to evolve biochemical systems with two optimal temperatures (Hochachka and Somero 1973). It is therefore not surprising that Gleeson (1979) found that "the adaptations to swimming have not resulted in increased transport costs on land" and that the aerobic scope is maximal at a body temperature of $35^{\circ} \mathrm{C}$ (Bennett et al. 1975), which is the temperature characteristic for socially or reproductively active marine iguanas.

Acknowledgements. We thank the Galapagos National Park and the Charles Darwin Station for permission to work and for their support. Special thanks are due to the Park Intendant Miguel Cifuentes and the Station's director Craig MacFarland. We appreciate the critique and suggestions that Drs. Catie Rechten, Volker Haas, Peter Vogel, Andrew Laurie, George Bartholomew and an anonymous reviewer contributed to this manuscript. This study was supported by the Max-Planck Gesellschaft and we thank Prof. Wolfgang Wickler for continuously supporting the work in Galapagos. B. Knauer prepared the figures and Lesley Gardiner and Phyllis Rechten corrected the English. This is contribution no. 384 of the Charles Darwin Foundation.

\section{References}

Auffenberg W (1972) Komodo Dragons. Nat Hist 81:52-59

Bartholomew GA (1966) A field study of temperature relations in the Galapagos marine iguana. Copeia $2: 241-250$

Bartholomew GA, Lasiewski RC (1965) Heating and cooling rates, heart rate and simulated diving in the Galapagos marine iguana. Comp Biochem Physiol 16:573-582

Bartholomew GA, Bennett AF, Dawson WR (1976) Swimming, diving and lactate production of the marine iguana, Amblyrhynchus cristatus. Copeia 1976:709-720

Bennett AF, Dawson WR, Bartholomew GA (1975) Effects of activity and temperature on aerobic and anaerobic metabolism in the Galapagos marine iguana. J Comp Physiol $100: 317-329$

Boersma PD (1982) The benefit of sleeping aggregations in 
marine iguanas, Amblyrhynchus cristatus. In: Burghardt GM, Rand AS (eds) Iguanas of the world. Noyes Publ., New Jersey, pp 292-300

Boersma PD (1984) An ecological study of the Galapagos marine iguana. In: Bowman RI, Berson M, Leviton AE (eds) Patterns of evolution in Galapagos organisms. Am Assoc Adv Sci Pac Div, San Francisco, pp 157-176

Carpenter CC (1966) The marine iguana of the Galapagos islands, its behavior and ecology. Proc Calif Acad Sci 34:329-376

Cott HB (1961) Scientific results of an inquiry into the ecology and economic status of the Nile Crocodile (Crocodilus niloticus) in Uganda and Northern Rhodesia. Trans Zool Soc London $29: 211-256$

Darwin C (1883) Journal of researches. New Edition, Appleton, $\mathrm{NY}$

Dawson WR, Bartholomew GA, Bennett AF (1977) A reappraisal of the aquatic specializations of the Galapagos marine iguana (Amblyrhynchus cristatus). Evolution 31:891-897

Eibl-Eibesfeldt I (1966) Das Verteidigen der Eiablageplätze bei der Hood Meerechse (Amblyrhynchus cristatus venustissimus). Z Tierpsychol 23:627-631

Gentry RL (1981) Northern fur seal Callorhinus ursinus (Linnaeus, 1758). In: Ridgway SH, Harrison RJ (eds) Handbook of marine mammals, vol 1. Academic Press, London, pp 143-160

Gleeson TT (1979) Foraging and transport costs in the Galapagos marine iguana, Amblyrhynchus cristatus. Physiol Zool $52: 549-557$

Hobson ES (1965) Observations on diving in Galapagos marine iguana, Amblyrhynchus cristatus (Bell). Copeia $1965: 249-250$

Hobson ES (1969) Remarks on aquatic habits of the Galapagos marine iguana, including submergence times, cleaning symbiosis, and the shark threat. Copeia 1969:401-402

Hochachka PW, Somero GN (1973) Strategies of biochemical adaptation. Sounders, Philadelphia

Nagy KA, Shoemaker VH (1984) Field energetics and food consumption of the Galapagos marine iguana, Amblyrhynchus cristatus. Physiol Zool 57:281-290

Newton I (1979) Population ecology of raptors. Poyser, Berkhamsted

Peters WD, Grubb TCJr (1983) An experimental analysis of sex-specific foraging in the downy woodpecker, Picoides pubescens. Ecology 64:1437-1443

Pond CM (1977) The significance of lactation in the evolution of mammals. Evolution $31: 177-199$

Pough HF (1973) Lizard energetics and diet. Ecology $54: 837-844$

Trillmich KGK (1983) The mating system of the marine iguana (Amblyrhynchus cristatus). Z Tierpsychol 63:141-172

Vleck D, Gleeson TT, Bartholomew GA (1981) Oxygen consumption during swimming in Galapagos marine iguanas and its ecological correlates. J Comp Physiol B $141: 531-536$

Werner EE, Gilliam JF (1984) The ontogenetic niche and species interactions in size-structured populations. Ann Rev Ecol Syst 15:393-425

White FN (1973) Temperature and the Galapagos marine iguana: Insights into reptilian thermoregulation. Comp Biochem Physiol 45:503-513 